\title{
Modelling and simulation of a magnetically coupled multiport dc-dc converter
}

\author{
Robert Griñó \\ Dept. of Automatic Control and \\ Inst. of Industrial and Control Engineering \\ Universitat Politècnica de Catalunya \\ roberto.grino@upc.edu
}

\author{
Arnau Dòria-Cerezo \\ Dept. of Electrical Engineering and \\ Inst. of Industrial and Control Engineering \\ Universitat Politècnica de Catalunya \\ arnau.doria@upc.edu
}

\begin{abstract}
This paper present two different approaches for modelling a magnetically coupled multiport dc-dc converter. First, from the magnetic coupling equations, the instantaneous dynamic model is obtained. Secondly, a behavioural dynamic model, based on averaging the power flowing among the ports, is derived. Some numerical simulations compare the temporal evolutions with the two obtained models.

Index Terms-Isolated multiport dc-dc converter, bidirectional power flow, high-frequency transformer, magnetic analysis, phase-shifted pulse width modulation.
\end{abstract}

\section{INTRODUCTION}

Magnetically coupled multiport dc-dc converters have recently attracted the interest of researchers for those applications connecting different dc networks. Examples include electric aircrafts [6], hybrid-electric vehicles [4], satellites [12], energy management systems [14], dc grids [10], ...

The attractiveness of this topology is, among others, related to the use of less components (because switching devices and storage elements are shared) resulting in lower overall mass and compact packaging, the power flow bidirectionality in each port, and the galvanic isolation among different dc networks thanks to the magnetic core. Usually the control of these converters is achieved by means of a high frequency square signal, where the control input is the phase-shift applied to each port, and under some conditions soft-switching can be achieved [11].

The particular case of dc-dc converters with two ports is known as dual active bridge (DAB) and was firstly studied in [2] [7]. For a multiport converters, several topologies have been proposed. Fully electrically isolated configurations, with a separate winding in each port, include single magnetic core [1] [3], a derived delta-type model for three-winding transformer [9], or dual-transformer topologies [5]. Other schemes reduce the number of windings (and, consequently, the size and weight), see [15]. In automotive applications, due to safety considerations, the fully isolated scheme is the preferred topology.

Usually, the design of control algorithms is based on a behavioural model obtained from averaging the power flow among different ports, and only few papers consider other approaches such as the generalized state-space averaging (GSSA) technique, see example in [8]. As far as our knowledge, the exact derivation of the behavioural model has not been completely reported in the literature assuming a periodic regime of voltages and currents in the high frequency transformer. Thus, this paper aims for developing two control-oriented models for a multiport dc-dc converters. Two different approaches are considered: i) a model obtained from the analysis of the magnetic coupling and, then, applying the Kirchoff Laws, ii) a model based on averaging the power flow among different ports.

The paper is organised as follows: Section II shows the basic scheme of the multiport dc-dc converter. The instantaneous model is developed in Section III, and the behavioural model is detailed in Section IV. Section V include numerical simulations comparing the two models. Finally, conclusions are stated in Section VI.

\section{THE MULTIPORT DC-DC CONVERTER}

Fig. 1 shows the schematic of the magnetically coupled multiport dc-dc converter. It consists of a $n$-port high frequency transformer with full-bridge converters, with a capacitor $C_{k}$ in their dc side, connected to each transformer winding.

\section{INSTANTANEOUS MODEL}

\section{A. Model of a multiport magnetic coupling}

Consider the multiport magnetic coupling shown in Fig. 2. From the Faraday's Law, the voltage across each $k$ winding is

$$
u_{k}(t)=\frac{\mathrm{d} \psi_{k}}{\mathrm{~d} t}
$$

where $\psi_{k}$ is the flux linkage, and $k \in\{1,2, \ldots, n\}$. The number of coils in a winding, $N_{k}$, relates the flux linkage with the magnetic flux, $\phi_{k}$, as

$$
\psi_{k}(t)=N_{k} \phi_{k}(t),
$$

and combining (1) and (2), one gets

$$
u_{k}(t)=N_{k} \frac{\mathrm{d} \phi_{k}}{\mathrm{~d} t} .
$$

The magnetic flux at each winding is

$$
\phi_{k}(t)=\phi(t)+\phi_{d k}(t)
$$




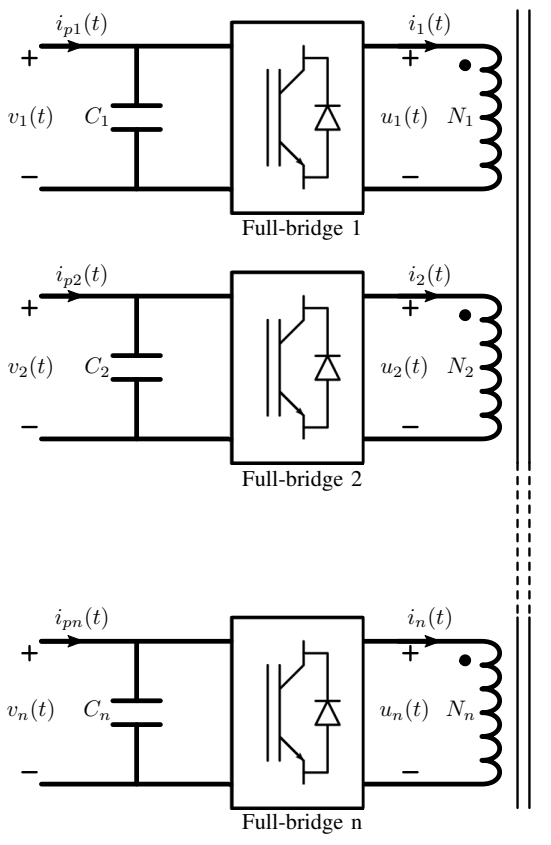

Fig. 1. Multiport dc-dc converter.

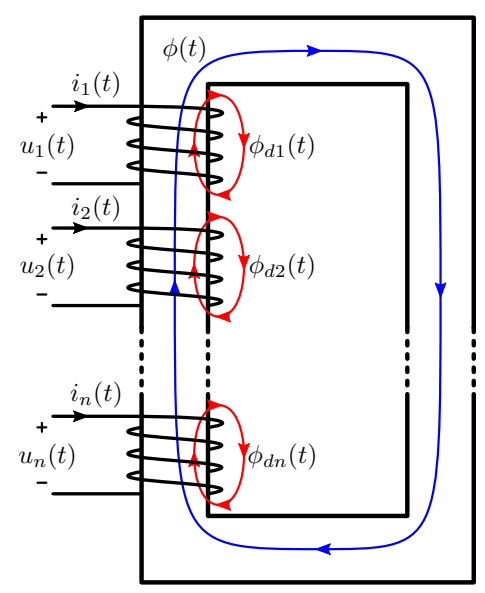

Fig. 2. Multiport magnetic coupling

with

$$
\begin{aligned}
\phi_{d k} & =\frac{N_{k}}{\mathcal{R}_{d k}} i_{k} \\
\phi & =\frac{\sum_{k=1}^{n} N_{k} i_{k}}{\mathcal{R}}
\end{aligned}
$$

where $\mathcal{R}$ and $\mathcal{R}_{d k}$ are the magnetic reluctances of the magnetic circuit and the magnetic leakage, respectively. Using (4)-(6) in (3), one gets

$$
u_{k}(t)=L_{k} \frac{\mathrm{d} i_{k}}{\mathrm{~d} t}+\sum_{l=1, l \neq k}^{n} M_{k l} \frac{\mathrm{d} i_{l}}{\mathrm{~d} t},
$$

where $L_{k}=M_{k}+L_{d k}, M_{k}=\frac{N_{k}^{2}}{\mathcal{R}}, L_{d k}=\frac{N_{k}^{2}}{\mathcal{R}_{d k}}$, and $M_{k l}=$ $M_{l k}=\frac{N_{k} N_{l}}{\mathcal{R}}$ for $k, l \in\{1,2, \ldots, n\}$ and $k \neq l$, are used.

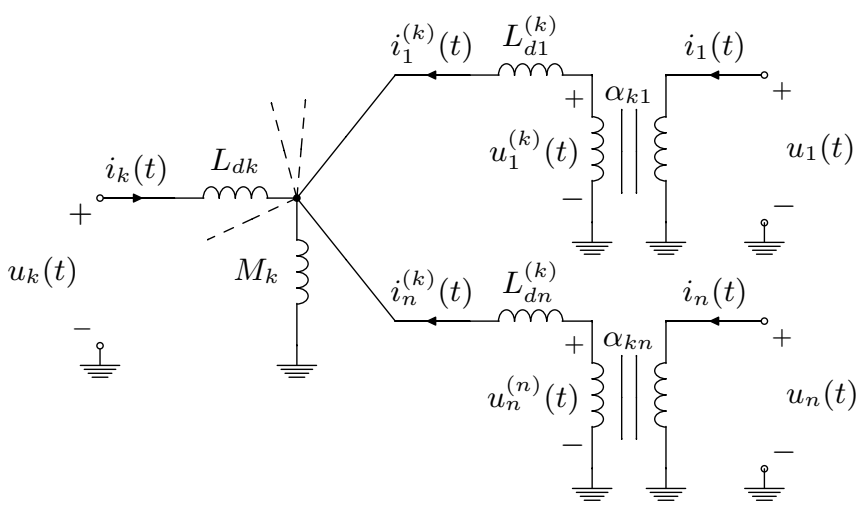

Fig. 3. Circuit scheme of a three-port transformer reduced to port $k$.

Finally, the magnetic coupling is represented in a compact form by

$$
M \frac{\mathrm{d} i}{\mathrm{~d} t}=u(t)
$$

where $i^{T}=\left(i_{1}(t), \ldots, i_{n}(t)\right), u^{T}=\left(u_{1}(t), \ldots, u_{n}(t)\right)$, and

$$
\boldsymbol{M}=\left(\begin{array}{cccc}
L_{1} & M_{12} & \ldots & M_{1 n} \\
M_{12} & L_{2} & \ldots & M_{2 n} \\
\vdots & \vdots & \ddots & \vdots \\
M_{1 n} & M_{2 n} & \ldots & L_{n}
\end{array}\right) .
$$

Remark 1: The transformer turn ratio of the $k$-th port with respect to the $l$-th port is defined as

$$
\alpha_{k l}=\frac{N_{l}}{N_{k}}, \quad k, l \in\{1,2, \ldots, n\}, \quad k \neq l .
$$

For the sake of simplicity, from now on, sometimes the time $(t)$ argument in the electrical variables is omitted.

\section{B. Magnetic coupling, referred to port $k$}

With the aim of simplifying the analysis, usually, transformers are referred to one port. This is equivalent to define new variables using the transformer ratio. Let us define the $l$-th voltage and current referred to port $k$ as

$$
\begin{aligned}
u_{l}^{(k)} & =\frac{N_{l}}{N_{k}} u_{l}=\alpha_{k l} u_{l}, \\
i_{l}^{(k)} & =\frac{N_{k}}{N_{l}} i_{l}=\frac{1}{\alpha_{k l}} i_{l},
\end{aligned}
$$

respectively. Then (7) results in

$$
\begin{aligned}
u_{k} & =L_{d k} \frac{\mathrm{d} i_{k}}{\mathrm{~d} t}+M_{k} \sum_{l=1}^{n} \frac{\mathrm{d} i_{l}^{(k)}}{\mathrm{d} t} \\
u_{l}^{(k)} & =L_{d l}^{(k)} \frac{\mathrm{d} i_{l}^{(k)}}{\mathrm{d} t}+M_{k} \sum_{m=1}^{n} \frac{\mathrm{d} i_{m}^{(k)}}{\mathrm{d} t}, \quad \forall l \neq k
\end{aligned}
$$

where $L_{d l}^{(k)}=\frac{N_{k}^{2}}{N_{l}^{2}} L_{d l}$. Fig. 3 shows the circuit scheme of a multiport magnetic coupling referred to port $k$. 


\section{Instantaneous model of a multiport $d c-d c$ converter}

The voltage applied to each $k$-winding of the magnetic coupling is, according the Kirchhoff Voltage Law,

$$
u_{k}=v_{k} \beta_{k}-r_{k} i_{k}
$$

where $v_{k}(t)$ is the voltage at the capacitor in the $k$-th port, $\beta_{k}(t)$ are the control signals (that take the discrete values $\beta_{k} \in$ $\{1,-1\})$, and $r_{k}$ is a parasitic resistance associated to the conduction losses of winding $k$.

On another hand, using the Kirchhoff Current Law, the capacitor dynamics is given by

$$
C_{k} \frac{\mathrm{d} v_{k}}{\mathrm{~d} t}=-i_{k} \beta_{k}+i_{p k}
$$

where $i_{p k}(t)$ is the current injected at port $k$.

Combining (8), (15) and (16), the dynamics of the dc-dc converter can be written in a compact form as,

$$
\begin{aligned}
& \boldsymbol{M} \frac{\mathrm{d} i}{\mathrm{~d} t}=-\boldsymbol{R} i+\beta \circ v \\
& \boldsymbol{C} \frac{\mathrm{d} v}{\mathrm{~d} t}=-\beta \circ i+i_{p}
\end{aligned}
$$

where $\circ$ is the Hadamard (or entrywise) product, the resistor and capacitor matrices are defined as $\boldsymbol{R}=\operatorname{diag}\left(r_{1}, \ldots, r_{n}\right)$ and $\boldsymbol{C}=\operatorname{diag}\left(C_{1}, \ldots, C_{n}\right)$, respectively, and the vectors $v^{T}=$ $\left(v_{1}, \ldots, v_{n}\right), i_{p}^{T}=\left(i_{p 1}, \ldots, i_{p n}\right), \beta^{T}=\left(\beta_{1}, \ldots, \beta_{n}\right)$.

Usually, a square wave signal is used as a modulation,

$$
\beta_{k}=\operatorname{sign}\left(\sin \left(\omega_{1} t-\theta_{k}\right)\right)
$$

where $\omega_{1}$ is the switching angular frequency (for control purposes, all ports have the same value) and $\theta_{k}$ is the shiftphase for the $k$-port that can be used as a control signal ${ }^{1}$.

\section{BEHAVIOURAL MODEL}

In this section, a model based on the power flow among the ports is considered. The model is obtained with the following assumptions:

A1 The magnetising inductances and the parasitic conduction losses are not considered $\left(M_{k l}=\infty\right.$ and $r_{k}=0$ for $k, l \in\{1,2, \ldots, n\}, k \neq l)$.

A2 The power transfer dynamics in the transformer is several orders of magnitude faster than the dynamics of the full converter (fixed by the values of the dc bus capacitors of the full-bridge converters). Thus, the power transfer in the high frequency transformer can be considered instantaneous and it can be modelled algebraically.

A3 Modulation signals, $\beta_{k}(t)$, are as in (19).

Under Assumptions A1 and A2, the dc bus voltages of the full-bridge converters can be assumed constant, i.e. $v_{k}(t)=$ $V_{k}, k \in\{1,2, \ldots, n\}$, and the full bridge operation generates

$$
{ }^{1} \theta_{k}=2 \pi \alpha \text { where } \alpha \in\left[-\frac{1}{2}, \frac{1}{2}\right]
$$

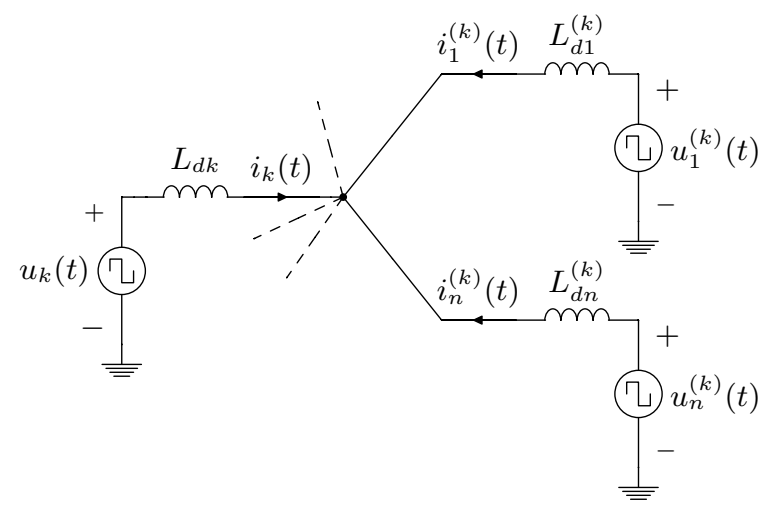

Fig. 4. Simplified circuit scheme (assuming $M_{k}=\infty$ ) of a three-port transformer reduced to port $k$.

periodic bipolar symmetric square wave voltages ${ }^{2}$ of constant amplitude, duty ratio equal to 0.5 and phase shift $\theta_{k}$. That is

$$
u_{k}(t)=V_{k} \operatorname{sign}\left(\sin \left(\omega_{1} t-\theta_{k}\right)\right)
$$

where $\omega_{1}=\frac{2 \pi}{T}$ is the fundamental frequency of the periodic voltages and currents in the transformer.

Notice that the voltages $u_{k}(t)$ at the transformer windings are square signals with zero mean and the control variables of the converter are, then, the relative phases between the voltages. The currents at the windings $i_{k}(t)$ have also zero mean. It is worth to remark that the power transfer in the transformer is done at the fundamental frequency of the voltage signals and their harmonics.

\section{A. Power flow analysis at a given port $k$}

Fig. 4 represents a multiport magnetic coupling referred to any port $k$, when applying the modulation signals (19) and the magnetising inductances are disregarded. Under a periodic regime, with (20), the Fourier series development of voltages in a multiport coupling is

$$
\begin{aligned}
u_{k}(t) & =\sum_{h=-\infty}^{\infty} c_{k, h} e^{j h \omega_{1} t} \\
u_{l}^{(k)}(t) & =\sum_{h=-\infty}^{\infty} c_{l, h}^{(k)} e^{j h \omega_{1} t}
\end{aligned}
$$

where $h \in \mathbb{Z}$, and

$$
\begin{aligned}
c_{k, h} & =\left\{\begin{array}{lll}
-j \frac{2 V_{k}}{h \pi} e^{-j h \theta_{k}}, & h & \bmod 2 \neq 0 \\
0, & h & \bmod 2=0
\end{array}\right. \\
c_{l, h}^{(k)} & =\left\{\begin{array}{lll}
-j \frac{2\left(V_{l} / \alpha_{k l}\right)}{h \pi} e^{-j h \theta_{l}}, & h & \bmod 2 \neq 0 \\
0, & h & \bmod 2=0
\end{array}\right.
\end{aligned}
$$

\footnotetext{
${ }^{2}$ These signals fulfil the property $u_{k}(t)=-u_{k}\left(t+\frac{T}{2}\right), \forall t$, with $T$ being the fundamental period of the signal. This property is equivalent to have, in their Fourier series developments, null harmonic content in the even harmonics including the zero one.
} 
Using the Rosen's Theorem [13], the nodal network in Fig. 4, is transformed into a meshed one where the linking inductance between port $k$ and any $l$-th port is given by

$$
L_{k l}^{(k)}=L_{d k} L_{d l}^{(k)} \sum_{m=1}^{n} \frac{1}{L_{d m}^{(k)}},
$$

and the (virtual) current flowing between ports $k$ and $l$ is

$$
i_{k l}(t)=\sum_{h=-\infty}^{\infty} d_{k l, h} e^{j h \omega_{1} t}
$$

where

$$
d_{k l, h}=\frac{c_{k, h}-c_{l, h}^{(k)}}{j h \omega_{1} L_{k l}^{(k)}} .
$$

The total averaged (active) power injected from port $k$, is

$$
P_{k}=\sum_{l=1, l \neq k}^{n} P_{k l}
$$

where ${ }^{3}$

$$
P_{k l}=\frac{1}{T} \int_{t-T}^{t} u_{k}(\tau) \overline{i_{k l}}(\tau) d \tau .
$$

Using (21) and (26)

$$
P_{k l}=c_{k, 0} d_{k l, 0}+2 \operatorname{Re}\left\{\sum_{h=1}^{\infty} c_{k, h} \overline{d_{k l, h}}\right\}
$$

that can be written as

$$
\begin{aligned}
P_{k l}= & -\frac{j 2 V_{k} V_{l}}{\alpha_{k l} \pi^{2} \omega_{1} L_{k l}^{(k)}}\left(\operatorname{Li}_{3}\left(e^{j \delta_{k l}}\right)-\operatorname{Li}_{3}\left(-e^{j \delta_{k l}}\right)\right. \\
& \left.-\operatorname{Li}_{3}\left(e^{-j \delta_{k l}}\right)+\operatorname{Li}_{3}\left(-e^{-j \delta_{k l}}\right)\right)
\end{aligned}
$$

where $\delta_{k l}=\theta_{l}-\theta_{k}$ and $\operatorname{Li}_{a}(z)=\sum_{r=1}^{\infty} \frac{z^{r}}{r^{a}}$ is the polylogarithm function. Fig. 5 shows graphically, in red, the relation among $P_{k l}$ and $\delta_{k l}$ for $\delta_{k l} \in[-\pi, \pi]$ that is equivalent to a temporal delay $\left[-\frac{T}{2}, \frac{T}{2}\right]$ between the voltages at the ports.

Developing the transferred power $P_{k l}$ in series around $\delta_{k l}=$ 0 results in

$$
P_{k l}=\frac{V_{k} V_{l}}{\alpha_{k l} \omega_{1} L_{k l}^{(k)}}\left(\delta_{k l}-\frac{\operatorname{sign}\left(\delta_{k l}\right) \delta_{k l}^{2}}{\pi}\right)+O\left(\delta_{k l}^{M}\right) .
$$

It is worth to remark that the order $M$ can be anyone and that the agreement between

$$
\begin{aligned}
P_{k l}^{\text {approx }} & =\frac{V_{k} V_{l}}{\alpha_{k l} \omega_{1} L_{k l}^{(k)}}\left(\delta_{k l}-\frac{\operatorname{sign}\left(\delta_{k l}\right) \delta_{k l}^{2}}{\pi}\right) \\
& =\frac{V_{k} V_{l}}{\alpha_{k l} \omega_{1} L_{k l}^{(k)}} \delta_{k l}\left(1-\frac{\left|\delta_{k l}\right|}{\pi}\right)
\end{aligned}
$$

and $P_{k l}$ in (31) is perfect in the interval $\delta_{k l} \in[-\pi, \pi]$, see Fig. 5. Equation (33) is the usually stated in the previous literature, e.g. [7]. Finally, the total power transferred to port $k$ results in

$$
P_{k}=\frac{V_{k}}{\omega_{1}} \sum_{l=1, l \neq k}^{n} \frac{V_{l}}{\alpha_{k l} L_{k l}^{(k)}} \delta_{k l}\left(1-\frac{\left|\delta_{k l}\right|}{\pi}\right) .
$$

\footnotetext{
$3 \bar{a}$ means the conjugate of the complex number $a$.
}

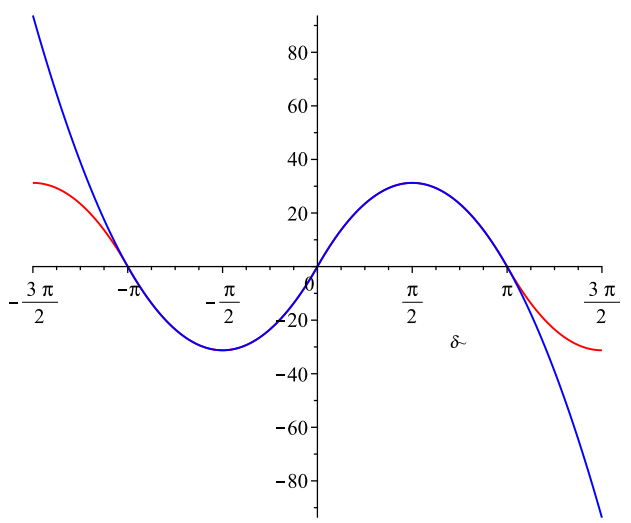

Fig. 5. Power transferred from ports $l$ to $k, P_{k l}$, with respect to $\delta_{k l}$. Exact relationship, eq. (31), in red, and approximation, eq. (33), in blue. The used parameters are $V_{k}=100 \mathrm{~V}, V_{l}=100 \mathrm{~V}, \omega_{1}=2 \pi 40.0 e 3 \mathrm{rad} / \mathrm{s}, L_{k l}=$ $1.0 e-3 \mathrm{H}$ and $\alpha_{k l}=1$.

B. On the approximations of $f(x)=x\left(1-\frac{|x|}{\pi}\right)$

This section discusses some approximations of $f(x)=$ $x\left(1-\frac{|x|}{\pi}\right)$ in order to eliminate the absolute value and obtain a simpler formulation to include it in the dynamic equations of the converter. Specifically, $f(x)$ is derivable at $x=0$ but it is only $\mathcal{C}^{1}$. Two possible approximations are

$$
\begin{aligned}
& f_{a}(x)=\frac{\pi}{4} \sin (x) \\
& f_{b}(x)=\frac{\pi / 4}{\tanh \left(\frac{a \pi}{2}\right)} \tanh (a x) .
\end{aligned}
$$

Approximation (35) is the most used in the literature, see example in [6], with a maximum absolute of $4.4 \%$ that occurs at $x= \pm 0.471972 \mathrm{rad}$. With this approximation the power transfer between ports $k$ and $l$ is

$$
P_{k l}^{\mathrm{a} 1}=\frac{V_{k} V_{l} \pi}{4 \alpha_{k l} \omega_{1} L_{k l}^{(k)}} \sin \left(\delta_{k l}\right)
$$

It is worth to note that this value is different from the one obtained taken into account only the fundamental components of square voltages in Equation (20). In this last case, the power transfer between ports $k$ and $l$ is

$$
P_{k l}^{\mathrm{a} 2}=\frac{8 V_{k} V_{l}}{\pi^{2} \alpha_{k l} \omega_{1} L_{k l}^{(k)}} \sin \left(\delta_{k l}\right) .
$$

Note that $\frac{P_{k l}^{\mathrm{a} 2}}{P_{k l}^{21}}=\frac{32}{\pi^{3}}=1.03205$.

On another hand, function (36) approximates better, for $a=1.1$ the maximum absolute error is $1.66 \%$ and it occurs at $x= \pm 1.234 \mathrm{rad}$, but is more complicated. See functions $f(x), f_{a}(x)$ and $f_{b}(x)$ in Fig. 6.

\section{Multiport dc-dc converter}

Similarly than Section III-C, the voltage at each port is given by the Kirchhoff Current Law and the capacitor dynamics,

$$
C_{k} \frac{\mathrm{d} v_{k}}{\mathrm{~d} t}=i_{p k}-\frac{1}{v_{k}} P_{k} .
$$




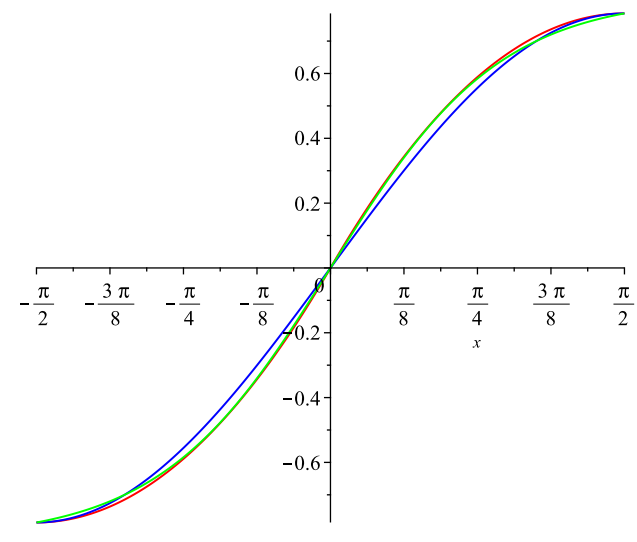

Fig. 6. $f(x)$ (red), $f_{a}(x)$ (blue) and $f_{b}(x)$ (green) vs $x \in\left[-\frac{\pi}{2}, \frac{\pi}{2}\right]$.

Finally, using (34), the voltage behaviour of each port can be modelled as

$$
C_{k} \frac{\mathrm{d} v_{k}}{\mathrm{~d} t}=i_{p k}-\frac{1}{\omega_{1}} \sum_{l=1, l \neq k}^{n} \frac{v_{l}}{\alpha_{k l} L_{k l}^{(k)}} \delta_{k l}\left(1-\frac{\left|\delta_{k l}\right|}{\pi}\right) .
$$

\section{COMParative Simulations: A THREe-PORT CONVERTER}

Let us consider a three dc bus system: a high voltage dc bus (nominal voltage $400 \mathrm{~V}$ ) and two low voltage buses (nominal voltages $48 \mathrm{~V}$ and $12 \mathrm{~V}$ ), with the parameters defined in Table I.

TABLE I

PARAMETERS OF THE SIMULATED THREE-PORT CONVERTER.

\begin{tabular}{||c|c||c|c||}
\hline$E_{1}$ & $400 \mathrm{~V}$ & $\omega_{1}$ & $2 \pi 40.0 e 3 \mathrm{rad} / \mathrm{s}$ \\
\hline$C_{2}$ & $600 \mu \mathrm{F}$ & $C_{3}$ & $200 \mu \mathrm{F}$ \\
\hline$\alpha_{12}$ & 0.12 & $L_{d 1}$ & $16.8 \mu \mathrm{H}$ \\
\hline$\alpha_{23}$ & 0.25 & $L_{d 2}$ & $0.994 \mu \mathrm{H}$ \\
\hline$\alpha_{13}$ & 0.03 & $L_{d 3}$ & $0.5 \mu \mathrm{H}$ \\
\hline$M_{1}$ & $280 \mu \mathrm{H}$ & $M_{12}$ & $336 \mu \mathrm{H}$ \\
\hline$M_{2}$ & $40.32 \mu \mathrm{H}$ & $M_{13}$ & $84 \mu \mathrm{H}$ \\
\hline$M_{3}$ & $2.52 \mu \mathrm{H}$ & $M_{23}$ & $10.08 \mu \mathrm{H}$ \\
\hline
\end{tabular}

The voltage at the high voltage bus is assumed constant, $E_{1}=400 \mathrm{~V}$, (mainly, due to the usual high capacity battery in these buses) and the two other buses have several loads connected to them. Let us assume that two resistive loads $\left(R_{2}, R_{3}\right)$ and two CPLs $\left(P_{p 2}, P_{p 3}\right)$ are connected to the low voltage buses ( $48 \mathrm{~V}$ and $12 \mathrm{~V}$ ), i.e.,

$$
\begin{aligned}
& i_{p 2}(t)=-\frac{v_{2}(t)}{R_{2}}-\frac{P_{p 2}}{v_{2}(t)} \\
& i_{p 3}(t)=-\frac{v_{3}(t)}{R_{3}}-\frac{P_{p 3}}{v_{3}(t)} .
\end{aligned}
$$

The modulation strategy is as in (19) setting $\theta_{1}=0$.
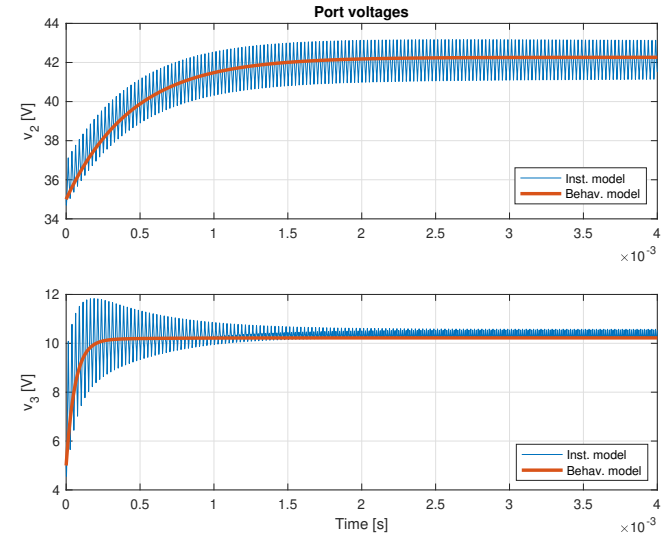

Fig. 7. Simulation results: Port voltages obtained from the instantaneous model (blue) and the behavioural model (red).

The model corresponding to the instantaneous behaviour obtained in Section III is

$$
\begin{aligned}
L_{1} \frac{\mathrm{d} i_{1}}{\mathrm{~d} t}+M_{12} \frac{\mathrm{d} i_{2}}{\mathrm{~d} t}+M_{13} \frac{\mathrm{d} i_{3}}{\mathrm{~d} t} & =E_{1} \beta_{1}-r_{1} i_{1} \\
M_{12} \frac{\mathrm{d} i_{1}}{\mathrm{~d} t}+L_{2} \frac{\mathrm{d} i_{2}}{\mathrm{~d} t}+M_{23} \frac{\mathrm{d} i_{3}}{\mathrm{~d} t} & =v_{2} \beta_{2}-r_{2} i_{2} \\
M_{13} \frac{\mathrm{d} i_{1}}{\mathrm{~d} t}+M_{23} \frac{\mathrm{d} i_{2}}{\mathrm{~d} t}+L_{3} \frac{\mathrm{d} i_{3}}{\mathrm{~d} t} & =v_{3} \beta_{3}-r_{3} i_{3} \\
C_{2} \frac{\mathrm{d} v_{2}}{\mathrm{~d} t} & =-i_{2} \beta_{2}-\frac{v_{2}}{R_{2}}-\frac{P_{p 2}}{v_{2}} \\
C_{3} \frac{\mathrm{d} v_{3}}{\mathrm{~d} t} & =-i_{3} \beta_{3}-\frac{v_{3}}{R_{3}}-\frac{P_{p 3}}{v_{3}}
\end{aligned}
$$

with

$$
\begin{aligned}
& \beta_{1}=\operatorname{sign}\left(\sin \left(\omega_{1} t\right)\right) \\
& \beta_{2}=\operatorname{sign}\left(\sin \left(\omega_{1} t-\theta_{2}\right)\right) \\
& \beta_{3}=\operatorname{sign}\left(\sin \left(\omega_{1} t-\theta_{3}\right)\right) .
\end{aligned}
$$

On another hand, from the analysis in Section IV, the behavioural model results in

$$
\begin{aligned}
C_{2} \frac{\mathrm{d} v_{2}}{\mathrm{~d} t} & =-\frac{v_{2}}{R_{2}}-\frac{P_{p 2}}{v_{2}}+\frac{E_{1}}{\alpha_{21} \omega_{1} L_{21}^{(2)}} \theta_{2}\left(1-\frac{\left|\theta_{2}\right|}{\pi}\right) \\
& -\frac{v_{3}}{\alpha_{23} \omega_{1} L_{23}^{(2)}}\left(\theta_{3}-\theta_{2}\right)\left(1-\frac{\left|\theta_{3}-\theta_{2}\right|}{\pi}\right) \\
C_{3} \frac{\mathrm{d} v_{3}}{\mathrm{~d} t} & =-\frac{v_{3}}{R_{3}}-\frac{P_{p 3}}{v_{3}}+\frac{E_{1}}{\alpha_{31} \omega_{1} L_{31}^{(3)}} \theta_{3}\left(1-\frac{\left|\theta_{3}\right|}{\pi}\right) \\
& +\frac{v_{2}}{\alpha_{32} \omega_{1} L_{32}^{(3)}}\left(\theta_{3}-\theta_{2}\right)\left(1-\frac{\left|\theta_{3}-\theta_{2}\right|}{\pi}\right) .
\end{aligned}
$$

Simulation tests using both models are performed using Matlab/Simulink. The test where: $P_{2}=P_{3}=0, R_{2}=0.75 \Omega$, $R_{3}=0.3 \Omega, \theta_{2}=25^{\circ}$ and $\theta_{3}=30^{\circ}$, with initial conditions: $i_{2}(0)=i_{3}(0)=0 \mathrm{~A}, v_{2}(0)=35 \mathrm{~V}$ and $v_{3}(0)=10 \mathrm{~V}$. For a fair comparison, parasitic resistances $r_{2}, r_{3}$ are neglected in 

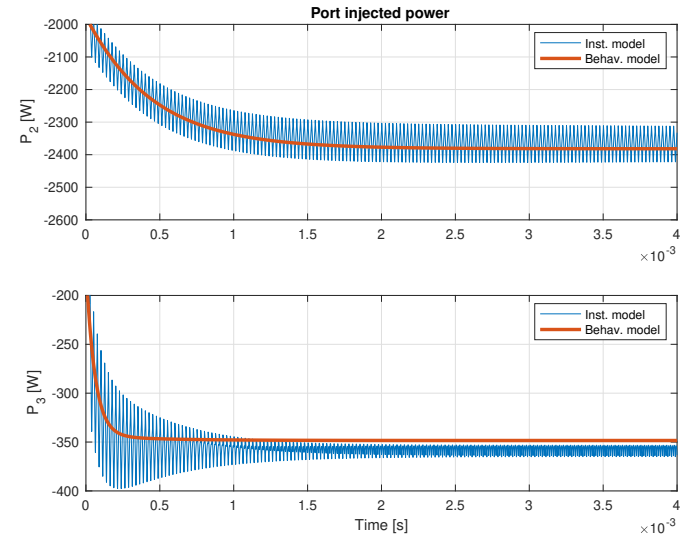

Fig. 8. Simulation results: Port injected power obtained from the instantaneous model (blue) and the behavioural model (red).
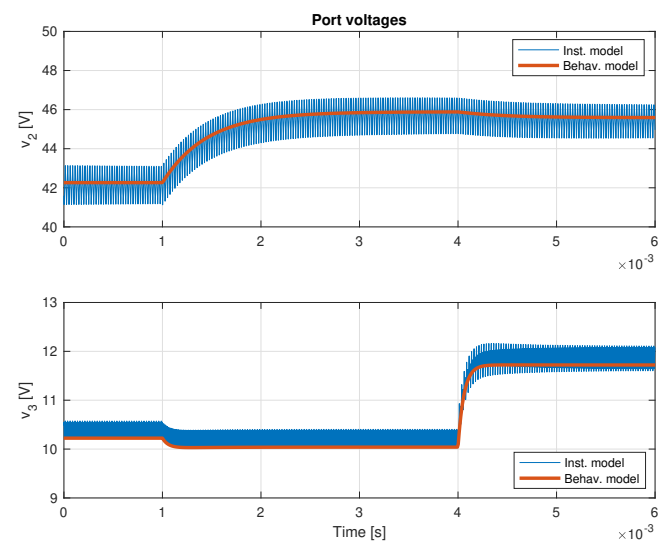

Fig. 9. Simulation results: Port voltages obtained from the instantaneous model (blue) and the behavioural model (red).

the instantaneous model. Fig. 7 and 8 show the voltages and powers, respectively.

A second test is performed starting at the final conditions of the previous test and changing the phase angles to $\theta_{2}=27.5^{\circ}$, $\theta_{3}=35^{\circ}$ at $t=1 \mathrm{~ms}$ and $t=4 \mathrm{~ms}$, respectively. Figures 9 and 10 show how the behaviour of model (51)-(52) approximates the one obtained with (43)-(47) for different values.

As expected, the behavioural model closely represents the averaged values (in red), while the model presented in Section III contains the switching behaviour. Some discrepancies are observed, specially in the power behaviour (Fig. 8 and 10) due to the assumptions done in Section IV. Is worth to mention that more differences are observed when the neglected effects (mainly parasitic resistances) are considered in the instantaneous model.

\section{CONCLUSIONS}

Two different models for a magnetically coupled multiport dc-dc converter are presented: an instantaneous model ob-
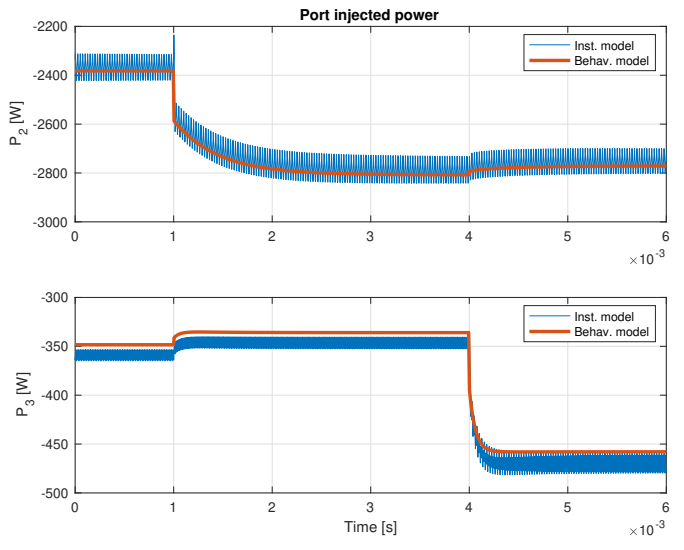

Fig. 10. Simulation results: Port injected power obtained from the instantaneous model (blue) and the behavioural model (red).

tained from the analysis of the magnetic coupling, and an averaged model based on the power flow among the ports. In all cases, the models are generalized for $n$ ports. In addition, conditions on the approximations used to derive the behavioural model are also discussed.

\section{ACKNOWLEDGMENT}

This work was partially supported by the Government of Spain through the Agencia Estatal de Investigación Project DPI2017-85404-P and by the Generalitat de Catalunya through the AGAUR Project 2017 SGR 872.

\section{REFERENCES}

[1] Y-M. Chen, Y-C. Liu, and F-Y. Wu. Multi-input DC/DC converter based on the multiwinding transformer for renewable energy applications. IEEE Trans. on Industry Applications, 38(4):1096-1104, 2002.

[2] R.W.A.A. De Doncker, D.M. Divan, and M.H. Kheraluwala. A threephase soft-switched high-power-density dc/dc converter for high-power applications. IEEE Trans. on Industry Applications, 27(1):63-73, 1991.

[3] J.L. Duarte, M. Hendrix, and M.G. Simoes. Three-port bidirectional converter for hybrid fuel cell systems. IEEE Trans. on Power Electronics, 22(2):480 - 487, 2007.

[4] B. Farhangi and H.A. Toliyat. Modeling and analyzing multiport isolation transformer capacitive components for onboard vehicular power conditioners. IEEE Trans. on Industrial Electronics, 62(5):3134-3142, 2015.

[5] V.N.S.R. Jakka, A. Shukla, and G.D. Demetriades. Dual-transformerbased asymmetrical triple-port active bridge (DT-ATAB) isolated DCDC converter. IEEE Trans. on Industrial Electronics, 64(6):4549-4560, 2017.

[6] B. Karanayil, M. Ciobotaru, and V.G. Agelidis. Power flow management of isolated multiport converter for more electric aircraft. IEEE Trans. on Power Electronics, 32(7):5850-5861, 2017.

[7] M.H. Kheraluwala, R.W. Gascoigne, D.M. Divan, and E.D. Baumann. Performance characterization of a high-power dual active bridge dc-todc converter. IEEE Trans. on Industry Applications, 28(6):1294-1301, 1992.

[8] Z. Li, Y. Wang, L. Shi, J. Huang, Y. Cui, and W. Lei. Generalized averaging modeling and control strategy for three-phase dual-activebridge DC-DC converters with three control variables. In Proc. IEEE Applied Power Electronics Conference and Exposition, 2017.

[9] D. Liu and H. Li. A ZVS bi-directional DC-DC converter for multiple energy storage elements. IEEE Trans. on Power Electronics, 21(5):15131517, 2006.

[10] H. Matsuo, W. Lin, F. Kurokawa, T. Shigemizu, and N. Watanabe. Characteristics of the multiple-input DC-DC converter. IEEE Trans. on Industrial Electronics, 51(3):625 - 631, 2004. 
[11] H. Pinheiro and P.K. Jain. Series-parallel resonant UPS with capacitive output DC bus filter for powering HFC networks. IEEE Trans. on Power Electronics, 17(6):971-979, 2002.

[12] Z. Qian, O. Abdel-Rahman, H. Al-Atrash, and I. Batarseh. Modeling and control of three-port DC/DC converter interface for satellite applications. IEEE Trans. on Power Electronics, 25(3):637-649, 2010.

[13] A. Rosen. A new network Theorem. Journal IEE, 62:916-918, 1924.

[14] H. Tao, J.L. Duarte, and M.A.M. Hendrix. Three-port triple-half-bridge bidirectional converter with zero-voltage switching. IEEE Trans. on Power Electronics, 23(2):782-792, 2008.

[15] C. Zhao, S.D. Round, and J.W. Kolar. An isolated three-port bidirectional DC-DC converter with decoupled power flow management. IEEE Trans. on Power Electronics, 23(5):2443-2453, 2008. 